Tohoku J. Exp. Med., 2010, 222, 1-5

Review

\title{
Prevalence, Classification, and Etiology of Pain in Parkinson's Disease: Association between Parkinson's Disease and Fibromyalgia or Chronic Widespread Pain
}

\author{
Katsuhiro Toda ${ }^{1}$ and Toshihide Harada ${ }^{2}$ \\ ${ }^{1}$ Department of Rehabilitation, Hatsukaichi Memorial Hospital, Hatsukaichi, Hiroshima, Japan \\ ${ }^{2}$ Department of Health Services Management, Faculty of Health and Welfare, Hiroshima International University, \\ Higashi-Hiroshima, Hiroshima, Japan
}

\begin{abstract}
Parkinson's disease (PD) is characterized by resting tremor, slow and decreased movement (hypokinesia and akinesia), rigidity, postural instability, problems with gait, and coordination. The prevalence of PD is between $0.1 \%$ and $0.3 \%$ in the general population and between $1 \%$ and $2 \%$ in persons 65 years of age or older. Patients with PD are more likely to suffer from pain. Indeed, the chief complaint of patients with severe motor disturbance and severe pain is pain rather than motor disturbance. Fibromyalgia (FM) is defined by widespread pain (pain in the left and right sides of the body, pain above the waist, pain below the waist, and axial skeletal pain) for more than 3 months and the presence of at least 11 of the 18 specified tender points. FM and chronic widespread pain (CWP), which is usually an incomplete form of $\mathrm{FM}$, cause pain in the musculoskeletal region, but their etiologies are unknown. Therefore, it is almost impossible to determine whether or not pain in the musculoskeletal region is in the musculoskeletal origin. We suspect that dysfunction or degeneration of the nerves that control pain, mind, and movement in the brain causes FM, depression, and PD, respectively. When pain in PD is discussed, FM and CWP should be considered because their prevalence is high. Patients with PD may be likely to suffer from FM and CWP; however, the prevalence of FM and CWP in patients with PD has not been reported. Here, we discuss the relationship between $\mathrm{PD}$ and FM or CWP.
\end{abstract}

Keywords: fibromyalgia; chronic widespread pain; Parkinson's disease; musculoskeletal pain; classification of pain Tohoku J. Exp. Med., 2010, 222 (1), 1-5. (C) 2010 Tohoku University Medical Press

The prevalence of Parkinson's disease (PD) is between $0.1 \%$ and $0.3 \%$ in the general population (Alves et al. 2008; Weintraub et al. 2008) and between $1 \%$ and $2 \%$ in persons 65 years of age or older (Alves et al. 2008; Weintraub et al. 2008). PD is characterized by resting tremor, slow and decreased movement (hypokinesia and akinesia), rigidity, postural instability, problems with gait, and impaired coordination. In addition to these motor symptoms, patients with PD often suffer from nonmotor symptoms, including severe pain (Riley et al. 1989; Clifford et al. 1998; Etchepare et al. 2006; Broetz et al. 2007; Defazio et al. 2008; Negre-Pages et al. 2008; Beiske et al. 2009).

Motor symptoms are easily perceived because they are visible. However, pain is not easily perceived because it is not visible. Modified Hoehn and Yahr staging is a commonly used clinical staging of PD. The staging is determined based on the severity of motor disturbance. Treatment outcomes are usually determined based on the severity of motor disturbance. However, pain aggravates quality of life as well as motor disturbance. Pain sometimes aggravates quality of life more than motor disturbance. In general, the chief complaint of patients with severe motor disturbance and severe pain is pain rather than motor disturbance. The previous sentence is easily understandable in patients with spinal cord injury. The same is true in patients with PD. If pain decreases, motor disturbance is often improved. Decreased pain directly improves quality of life and indirectly improves quality of life due to the improvement in motor disturbance.

Articles about pain in PD have been usually written by neurologists. The first author of this article is not a neurologist but specializes in chronic pain, especially fibromyalgia (FM) and chronic widespread pain (CWP). Neurologists are not so familiar with FM and CWP compared to rheumatologists or physicians who specialize in chronic pain. Therefore, articles about pain in PD did not refer to the relationship between PD and FM or CWP. When pain in PD is discussed, considering the possibility of FM and CWP is

Received November 16, 2009; revision accepted for publication August 4, 2010. doi: 10.1620/tjem.222.1

Correspondence: Katsuhiro Toda, M.D., Department of Rehabilitation, Hatsukaichi Memorial Hospital, 5-12 Youkoudai, Hatsukaichi-

City, Hiroshima 738-0060 Japan.

e-mail: goutattack@yahoo.co.jp 
essential, because the prevalence of FM and CWP is high. Here, we discuss the relationship between PD and FM or CWP.

\section{Fibromyalgia and chronic widespread pain}

FM is an important disorder in chronic pain. FM is defined by widespread pain (pain in the left side of the body, pain in the right side of the body, pain above the waist, pain below the waist, and axial skeletal pain) for more than 3 months and the presence of at least 11 of the 18 specified tender points (Wolfe et al. 1990). In addition to chronic pain and numbness, patients with FM frequently experience joint stiffness, fatigue, sleep disturbance, cognitive difficulties, anxiety, and depression. Some patients experience a number of other overlapping conditions, such as irritable bowel syndrome, tension-type headache, orofacial pain, vulvodynia, and interstitial cystitis. FM is predominant in women. Although the etiology of FM is not clear, the leading hypothesis is that pain stimulus from peripheral tissue causes central sensitization (Ablin et al. 2008). The hypothesis is that a lesion of the nerves that control pain in the brain causes pain. A lesion of the nerves that control mind in the brain causes depression and anxiety disorder. A lesion of the nerves that control movement in the brain causes PD (Fig. 1). Involvement of nerves that control movement in the brain and the nerves that control mind in the brain causes PD and depression. This is speculated to be one reason why patients with PD frequently suffer from depression. Forty-four percent of patients with PD were reported to suffer from depression (Shulman et al. 2002). Rates of depression are significantly higher among patients with FM and prevalence rates of major depressive disorder in FM patients range from 22 to $90 \%$ (Pae et al. 2008). If the hypothesis that a lesion of the nerves that control pain in the brain causes pain is correct, the involvement of the nerves that control movement in the brain and the nerves that control pain in the brain will also cause PD and pain. This is thought to be one reason why patients with PD frequently suffer from pain.

Broadly defined CWP is usually an unexplained disorder with widespread pain (pain in the left side of the body, pain in the right side of the body, pain above the waist, pain below the waist, and axial skeletal pain) for more than 3 months. Broadly defined CWP includes FM. Narrowly defined CWP, which is usually an incomplete form of FM, excludes FM. In this article, CWP usually means narrowlydefined CWP. Chronic regional pain (CRP) is an unexplained disorder that is similar to CWP but does not satisfy the diagnostic criteria of CWP. FM is thought to be at the extreme end in a continuum of CWP rather than a distinct medical entity (Forseth et al. 1999; Kosek 2006). CRP and CWP are thought to be a continuous disorder.

Although FM has objective signs of 11 or more tender points, CWP and CRP have no objective signs. If physicians do not know about FM, the tender points are not investigated. Even if 11 or more tender points exist, they are not objective signs which account for symptoms. If physicians do not know FM, CWP, and CRP, the disorders are likely to be diagnosed as psychogenic pain. If patients with CWP or CRP undergo FM treatment, the treatment outcome of CWP or CRP is better than the treatment outcome of FM. Treatment of neurogenic pain, such as FM, CWP, or CRP is completely different from the treatment of nociceptive pain (acute pain).

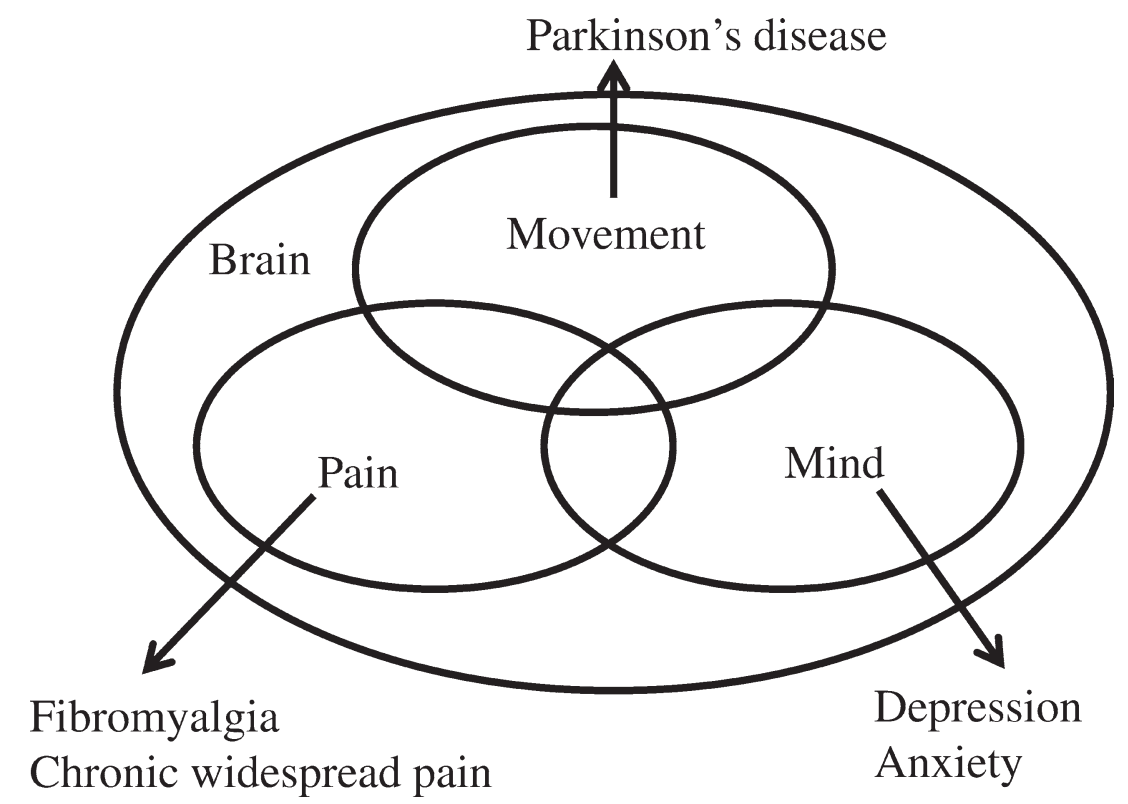

Fig. 1. Brain lesion and disorder (disease).

A lesion of the nerves that control pain in the brain is thought to cause fibromyalgia or chronic widespread pain. A lesion of the nerves that control mind in the brain causes depression and anxiety disorder. A lesion of the nerves that control movement in the brain causes Parkinson's disease. 


\section{Patients with Parkinson's disease are more likely to suffer from pain}

Do patients with PD suffer from pain more frequently than control groups? Beiske et al. (2009) reported that the PD patients experienced significantly more pain compared to the general population as measured by bodily pain in the Medical Outcomes Study 36-Items Short Form. Two studies showed that the frequency of pain in PD patients was not significantly greater than that in controls (Quittenbaum and Grahn 2004; Chaudhuri et al. 2006). On the other hand, 7 studies showed that the frequency of pain in PD patients was significantly greater than that in controls (Riley et al. 1989; Clifford et al. 1998; Etchepare et al. 2006; Broetz et al. 2007; Defazio et al. 2008; Negre-Pages et al. 2008; Beiske et al. 2009; Ehrt et al. 2009) (Table 1). Because there are more of the latter studies, including 2 extensive studies (Defazio et al. 2008; Negre-Pages et al. 2008), we think that the frequency of pain in PD patients is significantly greater than that in controls.

\section{Diagnosis of nonmotor presentations in PD is difficult}

Some patients with PD suffer from pain before motor symptoms are present. O'Sullivan et al. (2008) reported that $48(11.1 \%)$ out of 433 patients with PD had pain at presentation to their general practitioner. Nonmotor presentations in PD are initially frequently misdiagnosed, sometimes leading to inappropriate treatments, including surgery (O'Sullivan et al. 2008). Diagnosis of nonmotor presentations of PD is difficult. Especially, diagnosis of pain is difficult.

Unfortunately, pain in patients with PD are not properly treated (Sullivan et al. 2007). The reasons are as follows. 1 : Because pain is not visible, it is difficult for physicians to notice it. 2: Patients do not often mention pain. 3: Even if patients complain of pain, physicians do not often respond. 4: Physicians have knowledge of acute pain, but they usually do not have enough knowledge of chronic pain. Therefore, physicians often give acute pain treatment for chronic pain. 5: Pain without sufficient objective signs is often regarded as psychogenic pain. If patients are diagnosed with psychogenic pain, they usually cannot undergo appropriate medication.

\section{Classifications of pain in PD}

Many classifications of pain in PD have been advocated. There are two main types of pain classification based on the etiology of pain and the symptoms of pain. Because a mixed classification based on the etiology and symptoms causes confusion, a mix is not desirable. Classifications based on the etiology and symptoms should be described separately (Toda 2007b).

Pain defined based on the etiology of pain should be diagnosed based on the etiology of pain. Pain is classified into nociceptive, neurogenic, and psychogenic pain based on the etiology. Pain without sufficient objective signs is often regarded as psychogenic pain, whereas pain with sufficient objective signs is often regarded as neurogenic pain. Neurogenic and psychogenic pain are defined based on the etiology of pain; however, they are diagnosed based on pain symptoms (Toda 2007c), which is contradictory. If pain is defined based on the etiology, it should be diagnosed based on the etiology. Current medical practice cannot distinguish neurogenic pain from psychogenic pain based on the etiology of pain. We do not advocate that psychogenic pain is nonexistent. As previously explained, there are FM, CWP, and CRP. Before physicians diagnose psychogenic pain, they should suspect FM, CWP, or CRP. The diagnosis of FM, CWP, or CRP broadens treatment options, resulting in an improvement in treatment outcome.

There is a term 'musculoskeletal pain (MSP)'. The

Table 1. Comparison of pain between Parkinson's disease patients and control subjects.

\begin{tabular}{lccccl}
\hline \multicolumn{1}{c}{ Authors } & Year & \multicolumn{1}{c}{ PD } & Control & $P$ & \multicolumn{1}{c}{ Type of pain in each article } \\
\hline Quittenbaum & 2004 & $68.4(57)$ & $52.6(95)$ & 0.156 & Aching/pain last month \\
Chaudhuri & 2006 & $27.6(123)$ & $30.2(96)$ & 0.6 & Pains \\
\hline Riley & 1989 & $43.0(150)$ & $23.0(60)$ & $<0.01$ & Shoulder complaints \\
Riley & 1989 & $12.7(150)$ & $1.7(60)$ & $<0.02$ & Frozen shoulder \\
Clifford & 1998 & $23.4(115)^{\#}$ & & & Burning mouth \\
Etchepare & 2006 & $59.6(104)$ & $23.0(100)$ & $<0.0001$ & Back pain \\
Broetz & 2007 & $74.3(101)$ & $26.5(132)$ & $<0.0001$ & Back pain \\
Negre-Pages & 2008 & $61.8(450)$ & $58.2(98)$ & $0.51 *$ & Chronic pain \\
Defazio & 2008 & $69.9(402)$ & $62.8(317)$ & 0.04 & Pain for at least 3 months \\
Ehrt & 2009 & $67.0(227)$ & $39.0(100)$ & $<0.001$ & Pain \\
\hline
\end{tabular}

Numbers show percentages and parenthetic numbers show the number of subjects.

PD: Parkinson's disease

"Compared with the general population, PD patients were five times more likely to have burning mouth.

*After adjustment for osteo-articular comorbidity, PD patients were found to be twice as likely to suffer from chronic pain as patients with non-PD disorders $[\mathrm{OR}=1.9 ; 95 \%$ CI 1.2-3.2]. 
International Association for the Study of Pain did not define MSP in the Classification of Chronic Pain published in 1994 (Merskey and Bogduk 1994). Generally, low back pain is not pain of low back origin but pain in low back regardless of the location of a lesion. Similarly, MSP usually means pain in the musculoskeletal region; however, MSP sometimes means pain in the musculoskeletal region and it sometimes means pain caused by the musculoskeletal lesion, depending on the writer and reader. Ford used MSP to describe pain in the musculoskeletal region (Ford 1998). Beiske et al. (2009) defined MSP as pain due to parkinsonian rigidity, rheumatological disease or skeletal deformity, and therefore caused by the musculoskeletal lesion. If pain in the musculoskeletal region is expressed as pain in the musculoskeletal region and pain caused by a lesion in the musculoskeletal region is expressed as pain in the musculoskeletal origin, no confusion will occur.

Pain in the musculoskeletal origin is a term based on the etiology of pain. As mentioned above, pain in the musculoskeletal origin should be diagnosed based on the etiology of pain. It is easy to clarify the lesion location of nociceptive pain such as pain caused by a pulled muscle; however, it is difficult to clarify the lesion location of neurogenic pain. If the term 'pain in the musculoskeletal origin' is used, concrete diagnostic criteria should be used. For example, FM, which is neurogenic pain, causes pain in the musculoskeletal region, but the lesion location is not determined. It is usually impossible using current medical practice to decide whether or not pain in the musculoskeletal region is pain of musculoskeletal origin. Therefore, the term of "pain in the musculoskeletal origin" should not be used.

\section{Patients with Parkinson's disease may be more likely to suffer from fibromyalgia and chronic widespread pain}

The prevalence of FM is approximately $2 \%$ (Toda 2007a). The prevalence of CWP is reported to be between 5\% and 15\% (Croft et al. 1993; Hunt et al. 1999; Macfarlane et al. 1999; Benjamin et al. 2000; Macfarlane et al. 2001; Kato et al. 2006; McBeth and Jones 2007; Mas et al. 2008; Jones et al. 2009), but the prevalence of many studies is over 10\% (Croft et al. 1993; Macfarlane et al. 1999; Benjamin et al. 2000; Macfarlane et al. 2001; McBeth and Jones 2007; Jones et al. 2009). The prevalence of CRP is 1-2 times as frequent as that of CWP (Forseth et al. 1999; Bergman et al. 2001, 2002; Toda 2007a). Even if PD is unrelated to FM or CWP, the prevalence of FM and CWP in patients with PD is approximately $2 \%$ and $10 \%$, respectively. However, when pain in PD is discussed, FM and CWP are rarely considered. There is only one case report of a PD patient with FM (Toda et al. 2007d). FM and CWP should be considered in discussions of pain in PD. We speculate that the frequency of FM and CWP in PD patients is significantly greater than that in controls for the following reasons. First, patients with PD are more likely to suffer from depression and patients with FM are more likely to suffer from depression. Second, patients with PD are more likely to suffer from chronic pain. FM and CWP constitute a far greater percentage of chronic pain. Third, a lesion of the nerves that control movement in the brain causes PD. The hypothesis is that a lesion of the nerves that control pain in the brain causes FM. Involvement of the nerves that control movement in the brain and the nerves that control pain in the brain causes PD and FM or CWP

The prevalence of FM and CWP in patients with PD has not been reported. We would like physicians who treat PD to examine the prevalence of FM and CWP in patients with PD.

\section{Conclusion}

We suspect that lesions of the nerves that control pain, mind, and movement in the brain cause FM, depression, and $\mathrm{PD}$, respectively. We would like physicians who treat PD to think about pain, especially FM, CWP, and CRP. If physicians notice pain without objective signs, we would like them to suspect FM, CWP, or CRP. We would like physicians who treat PD to examine the prevalence of FM and CWP in patients with PD.

\section{References}

Ablin, J., Neumann, L. \& Buskila, D. (2008) Pathogenesis of fibromyalgia - a review. Joint Bone Spine, 75, 273-279.

Alves, G., Forsaa, E.B., Pedersen, K.F., Dreetz Gjerstad, M. \& Larsen, J.P. (2008) Epidemiology of Parkinson's disease. J. Neurol., 255 Suppl 5, 18-32.

Beiske, A.G., Loge, J.H., Ronningen, A. \& Svensson, E. (2009) Pain in Parkinson's disease: Prevalence and characteristics. Pain, 141, 173-177.

Benjamin, S., Morris, S., McBeth, J., Macfarlane, G.J. \& Silman, A.J. (2000) The association between chronic widespread pain and mental disorder: a population-based study. Arthritis Rheum., 43, 561-567.

Bergman, S., Herrstrom, P., Hogstrom, K., Petersson, I.F., Svensson, B. \& Jacobsson, L.T. (2001) Chronic musculoskeletal pain, prevalence rates, and sociodemographic associations in a Swedish population study. J. Rheumatol., 28, 1369-1377.

Bergman, S., Herrstrom, P., Jacobsson, L.T. \& Petersson, I.F. (2002) Chronic widespread pain: a three year followup of pain distribution and risk factors. J. Rheumatol., 29, 818-825.

Broetz, D., Eichner, M., Gasser, T., Weller, M. \& Steinbach, J.P. (2007) Radicular and nonradicular back pain in Parkinson's disease: a controlled study. Mov. Disord., 22, 853-856.

Chaudhuri, K.R., Martinez-Martin, P., Schapira, A.H., Stocchi, F., Sethi, K., Odin, P., Brown, R.G., Koller, W., Barone, P., MacPhee, G., Kelly, L., Rabey, M., MacMahon, D., Thomas, S., Ondo, W., Rye, D., Forbes, A., Tluk, S., Dhawan, V., Bowron, A., Williams, A.J. \& Olanow, C.W. (2006) International multicenter pilot study of the first comprehensive selfcompleted nonmotor symptoms questionnaire for Parkinson's disease: the NMSQuest study. Mov. Disord., 21, 916-923.

Clifford, T.J., Warsi, M.J., Burnett, C.A. \& Lamey, P.J. (1998) Burning mouth in Parkinson's disease sufferers. Gerodontology, 15, 73-78.

Croft, P., Rigby, A.S., Boswell, R., Schollum, J. \& Silman, A. (1993) The prevalence of chronic widespread pain in the general population. J. Rheumatol., 20, 710-713.

Defazio, G., Berardelli, A., Fabbrini, G., Martino, D., Fincati, E., Fiaschi, A., Moretto, G., Abbruzzese, G., Marchese, R., 
Bonuccelli, U., Del Dotto, P., Barone, P., De Vivo, E., Albanese, A., Antonini, A., Canesi, M., Lopiano, L., Zibetti, M., Nappi, G., Martignoni, E., Lamberti, P. \& Tinazzi, M. (2008) Pain as a nonmotor symptom of Parkinson disease: evidence from a case-control study. Arch. Neurol., 65, 11911194.

Ehrt, U., Larsen, J.P. \& Aarsland, D. (2009) Pain and its relationship to depression in Parkinson disease. Am. J. Geriatr. Psychiatry, 17, 269-275.

Etchepare, F., Rozenberg, S., Mirault, T., Bonnet, A.M., Lecorre, C., Agid, Y., Bourgeois, P. \& Fautrel, B. (2006) Back problems in Parkinson's disease: an underestimated problem. Joint Bone Spine, 73, 298-302.

Ford, B. (1998) Pain in Parkinson's disease. Clin. Neurosci., 5, 63-72.

Forseth, K.O., Forre, O. \& Gran, J.T. (1999) A 5.5 year prospective study of self-reported musculoskeletal pain and of fibromyalgia in a female population: significance and natural history. Clin. Rheumatol., 18, 114-121.

Hunt, I.M., Silman, A.J., Benjamin, S., McBeth, J. \& Macfarlane, G.J. (1999) The prevalence and associated features of chronic widespread pain in the community using the 'Manchester' definition of chronic widespread pain. Rheumatology (Oxford), 38, 275-279.

Jones, G.T., Power, C. \& Macfarlane, G.J. (2009) Adverse events in childhood and chronic widespread pain in adult life: Results from the 1958 British Birth Cohort Study. Pain, 143, 92-96.

Kato, K., Sullivan, P.F., Evengard, B. \& Pedersen, N.L. (2006) Chronic widespread pain and its comorbidities: a populationbased study. Arch. Intern. Med., 166, 1649-1654.

Kosek, E. (2006) Chapter 51 fibromyalgia. Handb. Clin. Neurol., 81, 763-777.

Macfarlane, G.J., McBeth, J. \& Silman, A.J. (2001) Widespread body pain and mortality: prospective population based study. $B M J, 323,662-665$.

Macfarlane, G.J., Morris, S., Hunt, I.M., Benjamin, S., McBeth, J., Papageorgiou, A.C. \& Silman, A.J. (1999) Chronic widespread pain in the community: the influence of psychological symptoms and mental disorder on healthcare seeking behavior. J. Rheumatol., 26, 413-419.

Mas, A.J., Carmona, L., Valverde, M. \& Ribas, B. (2008) Prevalence and impact of fibromyalgia on function and quality of life in individuals from the general population: results from a nationwide study in Spain. Clin. Exp. Rheumatol., 26, 519526.

McBeth, J. \& Jones, K. (2007) Epidemiology of chronic musculoskeletal pain. Best Pract. Res. Clin. Rheumatol., 21, 403-425.

Merskey, H. \& Bogduk, N. (1994) Classification of chronic pain, IASP Press, Seattle.
Negre-Pages, L., Regragui, W., Bouhassira, D., Grandjean, H. \& Rascol, O. (2008) Chronic pain in Parkinson's disease: the cross-sectional French DoPaMiP survey. Mov. Disord., 23, 1361-1369.

O’Sullivan, S.S., Williams, D.R., Gallagher, D.A., Massey, L.A., Silveira-Moriyama, L. \& Lees, A.J. (2008) Nonmotor symptoms as presenting complaints in Parkinson's disease: a clinicopathological study. Mov. Disord., 23, 101-106.

Pae, C.U., Luyten, P., Marks, D.M., Han, C., Park, S.H., Patkar, A.A., Masand, P.S. \& Van Houdenhove, B. (2008) The relationship between fibromyalgia and major depressive disorder: a comprehensive review. Curr. Med. Res. Opin., 24, 23592371.

Quittenbaum, B.H. \& Grahn, B. (2004) Quality of life and pain in Parkinson's disease: a controlled cross-sectional study. Parkinsonism Relat. Disord., 10, 129-136.

Riley, D., Lang, A.E., Blair, R.D., Birnbaum, A. \& Reid, B. (1989) Frozen shoulder and other shoulder disturbances in Parkinson's disease. J. Neurol. Neurosurg. Psychiatry, 52, 63-66.

Shulman, L.M., Taback, R.L., Rabinstein, A.A. \& Weiner, W.J. (2002) Non-recognition of depression and other non-motor symptoms in Parkinson's disease. Parkinsonism Relat. Disord., 8, 193-197.

Sullivan, K.L., Ward, C.L., Hauser, R.A. \& Zesiewicz, T.A. (2007) Prevalence and treatment of non-motor symptoms in Parkinson's disease. Parkinsonism Relat. Disord., 13, 545.

Toda, K. (2007a) The prevalence of fibromyalgia in Japanese workers. Scand. J. Rheumatol., 36, 140-144.

Toda, K. (2007b) Trigeminal neuralgia—symptom, diagnosis, classification, and related disorders- Oral Science International, 4, 1-9.

Toda, K. (2007c) The terms neurogenic pain and psychogenic pain complicate clinical practice. Clin. J. Pain, 23, 380-381.

Toda, K., Harada, T., Ishizaki, F., Horie, N. \& Yamada, T. (2007d) Parkinson disease patient with fibromyalgia: a case report. Parkinsonism Relat. Disord., 13, 312-314.

Weintraub, D., Comella, C.L. \& Horn, S. (2008) Parkinson's disease-Part 1: Pathophysiology, symptoms, burden, diagnosis, and assessment. Am. J. Manag. Care, 14, S40-48.

Wolfe, F., Smythe, H.A., Yunus, M.B., Bennett, R.M., Bombardier, C., Goldenberg, D.L., Tugwell, P., Campbell, S.M., Abeles, M., Clark, P., Fam, A.G., Farber, S.J., Fiechtner, J.J., Franklin, C.R., Gatter, R.A., Hamaty, D., Lessard, J., Lichtbroun, A.S., Masi, A.T., McCain, G.A., Reynolds, W.J., Romano, T.J., Russell, I.J. \& Sheon, R.P. (1990) The American College of Rheumatology 1990 Criteria for the Classification of Fibromyalgia. Report of the Multicenter Criteria Committee. Arthritis Rheum, 33, 160-172. 